\title{
Celestial current algebra from Low's subleading soft theorem
}

\author{
Elizabeth Himwich ${ }^{*}$ and Andrew Strominger ${ }^{\dagger}$ \\ Center for the Fundamental Laws of Nature, Harvard University, Cambridge, Massachusetts 02138, USA
}

(Received 7 August 2019; published 3 September 2019)

\begin{abstract}
The leading soft photon theorem implies that four-dimensional scattering amplitudes are controlled by a two-dimensional (2D) $U(1)$ Kac-Moody symmetry that acts on the celestial sphere at null infinity $(\mathcal{I})$. This celestial $U(1)$ current is realized by components of the electromagnetic vector potential on the boundaries of $\mathcal{I}$. Here, we develop a parallel story for Low's subleading soft photon theorem. It gives rise to a second celestial current, which is realized by vector potential components that are subleading in the large radius expansion about the boundaries of $\mathcal{I}$. The subleading soft photon theorem is reexpressed as a celestial Ward identity for this second current, which involves novel shifts by one unit in the conformal dimension of charged operators.
\end{abstract}

DOI: 10.1103/PhysRevD.100.065001

\section{INTRODUCTION}

In any four-dimensional (4D) theory with photons, the soft photon theorem implies [1-5] the existence of a two-dimensional (2D) $U(1)$ Kac-Moody symmetry. The consequences of the symmetry become most transparent when 4D scattering amplitudes are reexpressed as correlation functions on the celestial sphere at null infinity $(\mathcal{I})$, on which the 4D Lorentz group acts as the 2D Euclidean conformal group. The Kac-Moody currents act on this celestial sphere and are sourced by electromagnetic charge currents that cross it. All amplitudes are thereby highly constrained, and in particular are set to zero by infrared divergences [6] if the associated conservation laws are violated. The celestial Kac-Moody current may be explicitly realized by a sum of the gauge potentials on the $S^{2}$ boundaries of $\mathcal{I}$, denoted $A_{\bar{z}}^{(0)}$ below. This story is reviewed in [7].

In the 1950s Low and others [8-12] established a second, universal, relation governing the subleading term in the soft expansion of an asymptotic photon. A similar story is expected to derive from this universal relation, but so far is only partially understood [13-15].

In this paper we show that the subleading soft theorem implies a second current algebra on the celestial sphere. The currents are the constructed from the boundary values of the subleading term of the gauge potential,

\footnotetext{
*himwich@g.harvard.edu

†strominger@physics.harvard.edu
}

Published by the American Physical Society under the terms of the Creative Commons Attribution 4.0 International license. Further distribution of this work must maintain attribution to the author(s) and the published article's title, journal citation, and DOI. Funded by SCOAP . denoted $A_{\bar{z}}^{(1)}$, in the large radius expansion around $\mathcal{I}$. Naively, $A_{\bar{z}}^{(1)}$ is determined from the leading potential $A_{\bar{z}}^{(0)}$ by the equations of motion and is not an independent field. However, in attempting to explicitly solve for $A_{\bar{z}}^{(1)}$ in terms of $A_{\bar{z}}^{(0)}$, one encounters an integration function on the sphere. This implies that the boundary values of $A_{\bar{z}}^{(1)}$ are independent fields after all, and in fact turn out to comprise an independent "subleading" current algebra.

The current algebra generated on the celestial sphere by boundary values of $A_{\bar{z}}^{(1)}$ has interesting and unconventional features. The operator product expansion of the subleading current with a charged operator with $2 \mathrm{D}$ conformal weights $(h, \bar{h})$ shifts the weights to $\left(h-\frac{1}{2}, \bar{h}-\frac{1}{2}\right)$. This is possible because such operators lie in the continuous unitary principal series. Our main result is formula (26) below which describes this action. It will be interesting to eventually understand the constraints of (26) on scattering amplitudes.

In this paper we make the simplifying restrictions that there are no long range magnetic fields near spatial infinity and that charge is carried by massless scalar fields. As discussed in the text, we expect our results to hold in a more general context, as their form is largely dictated by symmetries.

This note is organized as follows. In Sec. II, we introduce our conventions and present basic formulas. In Sec. III, we rewrite the subleading soft theorem as a relation between the boundary values of a subleading gauge parameter. In Sec. IV, we take the quantum matrix element of this conservation law and express it as a Ward identity for a novel 2D current algebra on the celestial sphere. The Appendix gives some details of the asymptotic expansion about $\mathcal{I}$ in Lorenz gauge. 


\section{MAXWELL EQUATIONS IN LORENZ GAUGE}

We largely employ the retarded (advanced) coordinates on flat Minkowski space

$$
\begin{aligned}
d s^{2} & =-d u^{2}-2 d u d r+2 r^{2} \gamma_{z \bar{z}} d z d \bar{z} \\
& =-d v^{2}+2 d v d r+2 r^{2} \gamma_{z \bar{z}} d z d \bar{z}
\end{aligned}
$$

with $u(v)$ retarded (advanced) time and $\gamma_{z \bar{z}}=2 /(1+z \bar{z})^{2}$ the unit round metric on $S^{2}$. These are related to the Cartesian coordinates $\left(x^{0}, x^{1}, x^{2}, x^{3}\right)$ by

$$
\begin{aligned}
x^{0} & =u+r=v-r \\
x^{1}+i x^{2} & =\frac{2 r z}{1+z \bar{z}} \\
x^{3} & =\frac{r(1-z \bar{z})}{1+z \bar{z}} .
\end{aligned}
$$

In this paper we use the Lorenz gauge condition $\nabla_{\mu} A^{\mu}=0$. The Maxwell equations $\nabla_{\mu} F^{\mu \nu}=e^{2} j^{\nu}$ in this gauge in retarded coordinates are

$$
\begin{aligned}
2 r \partial_{r}\left(r \partial_{u} A_{u}\right)-\partial_{r}\left(r^{2} \partial_{r} A_{u}\right)-2 \gamma^{z \bar{z}} \partial_{z} \partial_{\bar{z}} A_{u} & =e^{2} r^{2} j_{u} \\
2 \partial_{r}\left(r A_{u}\right)+2 \partial_{u} \partial_{r}\left(r^{2} A_{r}\right)-\partial_{r}^{2}\left(r^{2} A_{r}\right)-2 \gamma^{z \bar{z}} \partial_{z} \partial_{\bar{z}} A_{r} & =e^{2} r^{2} j_{r} \\
-2 r^{2} \partial_{u} \partial_{r} A_{z}+r^{2} \partial_{r}^{2} A_{z}-2 r \partial_{z}\left(A_{r}-A_{u}\right)+2 \partial_{z}\left(\gamma^{z \bar{z}} \partial_{\bar{z}} A_{z}\right) & =e^{2} r^{2} j_{z} .
\end{aligned}
$$

See Appendix for further details.

\section{SUBLEADING SOFT THEOREM AS SUBLEADING GAUGE TRANSFORMATION}

Low's subleading soft photon theorem, following the notation of [13], can be written as an asymptotic symmetry acting on in- and out-states. Denote a state with $n$ massless hard particles of energies $\omega_{k}$, charges $e Q_{k}$ and momenta

$$
p_{k}^{\mu}=\frac{\omega_{k}}{1+z_{k} \bar{z}_{k}}\left(1+z_{k} \bar{z}_{k}, z_{k}+\bar{z}_{k}, i\left(\bar{z}_{k}-z_{k}\right), 1-z_{k} \bar{z}_{k}\right)
$$

by $\left|z_{1}, \ldots\right\rangle$ and hard $S$-matrix elements by $\left\langle z_{n+1}, \ldots\right.$ $\left.|\mathcal{S}| z_{1}, \ldots\right\rangle$. The Low-Burnett-Kroll-Goldberger-Gell-Mann soft theorem says that if a positive helicity photon with energy $\omega \rightarrow 0$, the first two terms of the soft expansion are

$$
\begin{aligned}
& \left\langle z_{n+1}, \ldots\left|a_{-}^{\text {out }}(\vec{q}) \mathcal{S}\right| z_{1}, \ldots\right\rangle \\
& \quad=\left(J^{(0)-}+J^{(1)-}\right)\left\langle z_{n+1}, \ldots|\mathcal{S}| z_{1}, \ldots\right\rangle,
\end{aligned}
$$

with

$$
\begin{aligned}
& J^{(0)-}=e \sum_{k} Q_{k} \frac{p_{k} \cdot \varepsilon^{-}}{p_{k} \cdot q} \sim \mathcal{O}\left(\omega^{-1}\right), \\
& J^{(1)-}=-i e \sum_{k} Q_{k} \frac{q_{\mu} \varepsilon_{\nu}^{-} J_{k}^{\mu \nu}}{p_{k} \cdot q} \sim \mathcal{O}\left(\omega^{(0)}\right)
\end{aligned}
$$

with $\varepsilon$ the photon polarization and $J_{k}^{\mu \nu}$ the total angular momentum of the $k$ th particle. The $J^{(0)}$ - contribution can be eliminated with the projection operator $\left(1+\omega \partial_{\omega}\right)$. In [13] it was shown that, for the special case of a scalar field [16] with $J_{k \mu \nu}=-i\left(p_{k \mu} \frac{\partial}{\partial p_{k}^{\nu}}-p_{k \nu} \frac{\partial}{\partial p_{k}^{\mu}}\right)$, rewriting $\left(p_{k}^{\mu}, q^{\mu}\right)$ in terms of $\left(E_{k}, z_{k}, \bar{z}_{k}\right)$ gives

$$
\begin{aligned}
\lim _{\omega \rightarrow 0}(1 & \left.+\omega \partial_{\omega}\right)\left\langle z_{n+1}, \ldots\left|a_{-}^{\text {out }}(\vec{q}) \mathcal{S}\right| z_{1}, \ldots\right\rangle \\
= & -e \sum_{k} \frac{Q_{k}}{\sqrt{2}\left(\bar{z}_{k}-\bar{z}\right)}\left[\left(1+z \bar{z}_{k}\right)\right) \partial_{E_{k}} \\
& \left.+\frac{1}{E_{k}}\left(z-z_{k}\right)\left(1+z_{k} \bar{z}_{k}\right) \partial_{z_{k}}\right]\left\langle z_{n+1}, \ldots|\mathcal{S}| z_{1}, \ldots\right\rangle .
\end{aligned}
$$

As in [13] it is useful to define operators $F_{u \bar{z}}^{\text {sub }}$ that create subleading soft photons. These are defined on $\mathcal{I}^{+}$in terms of the photon polarization

$$
\hat{\varepsilon}_{\bar{z}}^{+}=\frac{\partial_{\bar{z}} x^{\mu}}{r} \varepsilon_{\mu}^{+}=\frac{\sqrt{2}}{1+z \bar{z}}
$$

by

$$
\begin{aligned}
F_{u \bar{z}}^{\text {sub }} & :=\int d u u F_{u \bar{z}}^{(0)}=\int d u u \partial_{u} A_{\bar{z}}^{(0)} \\
& =\frac{i e}{8 \pi} \hat{\varepsilon}_{\bar{z}}^{+} \lim _{\omega \rightarrow 0}\left(1+\omega \partial_{\omega}\right)\left[a_{-}^{\text {out }}(\omega \hat{x})-a_{+}^{\text {out }}(\omega \hat{x})^{\dagger}\right] .
\end{aligned}
$$

The fields in this expression, and subsequent expressions, are the functions of $(u, z, \bar{z})$ that appear as coefficients in the asymptotic $\frac{1}{r}$ expansion about $\mathcal{I}^{+}$. The order $\frac{1}{r^{n}}$ at which they appears in this expansion is denoted by the superscript $(n)$. For simplicity, we restrict here to the case where there are no long range magnetic fields near spatial infinity so that $A_{z}^{(0)}$ is pure gauge and $F_{z \bar{z}}^{(0)}=0$ at $\mathcal{I}_{ \pm}^{+}$. This allows us to focus clearly on the difference in boundary values of pure gauge part of the photon gauge field, which is the quantity of physical interest in this note. Multiplying (7) by $\hat{\varepsilon}_{\bar{z}}^{+}$and then acting with $\gamma^{z \bar{z}} D_{\bar{z}} D_{z}$ and using (9) gives 


$$
\begin{aligned}
\left\langle z_{n+1}, \ldots\left|Q_{S}^{+} \mathcal{S}\right| z_{1}, \ldots\right\rangle & \\
= & -\frac{i}{4 \pi} \sum_{k} Q_{k}\left[-2 \pi \gamma^{z \bar{z}} D_{\bar{z}} \delta^{2}\left(z-z_{k}\right) \partial_{E_{k}}\right. \\
& \left.+\frac{1}{E_{k}} \frac{\gamma^{z_{k} \bar{z}_{k}}}{\left(\bar{z}-\bar{z}_{k}\right)^{2}} \partial_{z_{k}}\right]\left\langle z_{n+1}, \ldots|\mathcal{S}| z_{1}, \ldots\right\rangle,
\end{aligned}
$$

where we can define the "soft" charge

$$
Q_{S}^{+}=\frac{2}{e^{2}} \int d u и \partial_{u} \gamma^{z \bar{z}} D_{\bar{z}} D_{z} A_{\bar{z}}^{(0)} .
$$

For the leading soft charge, the analog of the soft term is a total $u$-derivative and reduces to a difference between two terms on the boundaries of $\mathcal{I}^{+}$, signaling the central role of $\mathcal{I}$ boundary dynamics. In contrast, this total derivative structure is not manifest in the soft term given in [13] and in (11). However, we now show that this structure reappears when $Q_{S}^{+}$is reexpressed in terms of the subleading component $A_{z}^{(1)}$ of the gauge field, which enables one to rewrite it in terms of hard currents and the $\mathcal{I}_{ \pm}^{+}$boundary values of $A_{z}^{(1)}$. The elimination of $A_{\bar{z}}^{(0)}$ from (11) in favor of $A_{\bar{z}}^{(1)}$ proceeds via the asymptotic expansion of the Maxwell equations, which are without sources for the soft insertion (see Appendix for details)

$$
\partial_{u}^{2} A_{\bar{z}}^{(1)}=-\partial_{u} D^{z} D_{z} A_{\bar{z}}^{(0)} .
$$

This allows us to rewrite the soft charge as

$$
Q_{S}^{+}=\left.\frac{2}{e^{2}}\left(1-u \partial_{u}\right) A_{\bar{z}}^{(1)}\right|_{\mathcal{I}_{ \pm}^{+}} ^{\mathcal{I}_{+}^{+}} .
$$

Lorenz gauge $\nabla^{\mu} A_{\mu}=0$ leaves unfixed residual gauge transformations of the form $A_{\mu} \rightarrow A_{\mu}+\partial_{\mu} \varepsilon$ with $\square \varepsilon=0$. The solution to this equation in retarded coordinates requires two pieces of free data, at different orders in the asymptotic expansion: the free function $\varepsilon^{(0)}(z, \bar{z})$, which is related to the leading soft theorem, and the free function $\varepsilon^{(1)}(u, z, \bar{z})$, which is independent free data. This latter residual freedom enables us to fix the subsidiary gauge condition

$$
A_{u}^{(1)}=0,
$$

which implies that $\partial_{u} \varepsilon^{(1)}=0$. We are left with a free function $\varepsilon^{(1)}(z, \bar{z})$. The gauge transformations are parametrized as

$\varepsilon=\varepsilon^{(0)}(z, \bar{z})+\frac{u}{2} D^{2} \varepsilon^{(0)}(z, \bar{z}) \frac{\log r}{r}+\frac{\varepsilon^{(1)}(z, \bar{z})}{r}+\cdots$
At early and late times along future null infinity, where the matter current is zero, the field configurations return to pure gauge. Hence the asymptotic behavior near $\mathcal{I}_{ \pm}^{+}$is

$$
\begin{gathered}
A_{\bar{z} \pm}^{(0)}=D_{\bar{z}} \varphi_{ \pm}^{(0)}(z, \bar{z}), \quad \tilde{A}_{\bar{z} \pm}^{(1)}=\frac{u}{2} D_{\bar{z}} D^{2} \varphi_{ \pm}^{(0)}(z, \bar{z}), \\
A_{\bar{z} \pm}^{(1)}=D_{\bar{z}} \varphi_{ \pm}^{(1)}(z, \bar{z}),
\end{gathered}
$$

where the tilde denotes a $\log r$ dependence (see Appendix for details) and where the boundary fields $\varphi$ shift under gauge transformations as $\varphi_{ \pm}^{(0)} \rightarrow \varphi_{ \pm}^{(0)}+\varepsilon^{(0)}$ and $\varphi_{ \pm}^{(1)} \rightarrow$ $\varphi_{ \pm}^{(1)}+\varepsilon^{(1)}$. The difference in their values at $\mathcal{I}_{+}^{+}$and $\mathcal{I}_{-}^{+}$is determined by the action of the soft factor and cannot be gauge-fixed to zero. To underscore this, we rewrite (10) as

$$
\begin{aligned}
\left\langle z_{n+1}, \ldots\right| & \left(\frac{2}{e^{2}} D_{\bar{z}} \varphi^{(1)} \mid \begin{array}{l}
\mathcal{I}_{+}^{+} \\
\mathcal{I}_{-}^{+}
\end{array}\right) \mathcal{S}\left|z_{1}, \ldots\right\rangle \\
= & -\frac{i}{4 \pi} \sum_{k} Q_{k}\left[-2 \pi \gamma^{z \bar{z}} D_{\bar{z}} \delta^{2}\left(z-z_{k}\right) \partial_{E_{k}}\right. \\
& \left.+\frac{1}{E_{k}} \frac{\gamma^{z_{k} \bar{z}_{k}}}{\left(\bar{z}-\bar{z}_{k}\right)^{2}} \partial_{z_{k}}\right]\left\langle z_{n+1}, \ldots|\mathcal{S}| z_{1}, \ldots\right\rangle .
\end{aligned}
$$

Similarly, for the insertion of an incoming soft photon $\mathcal{I}^{-}$,

$$
\begin{aligned}
\left\langle z_{n+1}, \ldots\left|\mathcal{S}\left(\left.\frac{2}{e^{2}} D_{\bar{z}} \varphi^{(1)}\right|_{\mathcal{I}_{-}^{-}} ^{\mathcal{I}_{+}^{-}}\right)\right| z_{1}, \ldots\right\rangle \\
=\frac{i}{4 \pi} \sum_{k} Q_{k}\left[-2 \pi \gamma^{z \bar{z}} D_{\bar{z}} \delta^{2}\left(z-z_{k}\right) \partial_{E_{k}}\right. \\
\left.\quad+\frac{1}{E_{k}} \frac{\gamma^{z_{k} \bar{z}_{k}}}{\left(\bar{z}-\bar{z}_{k}\right)^{2}} \partial_{z_{k}}\right]\left\langle z_{n+1}, \ldots|\mathcal{S}| z_{1}, \ldots\right\rangle .
\end{aligned}
$$

To write a shift along all of $\mathcal{I}$, we consider

$$
\begin{aligned}
\left\langle z_{n}, \ldots\left|Q_{S}^{+} \mathcal{S}-\mathcal{S} Q_{S}^{-}\right| z_{1}, \ldots\right\rangle & \\
= & -\frac{i}{2 \pi} \sum_{k} Q_{k}\left[-2 \pi \gamma^{z \bar{z}} D_{\bar{z}} \delta^{2}\left(z-z_{k}\right) \partial_{E_{k}}\right. \\
& \left.+\frac{1}{E_{k}} \frac{\gamma^{z_{k} \bar{z}_{k}}}{\left(\bar{z}-\bar{z}_{k}\right)^{2}} \partial_{z_{k}}\right]\left\langle z_{n+1}, \ldots|\mathcal{S}| z_{1}, \ldots\right\rangle .
\end{aligned}
$$

We see that, if there is a nontrivial scattering process, it is impossible to set $A_{z}^{(1)}=D_{z} \varphi^{(1)}$ to zero on all boundaries of $\mathcal{I}$, just as the leading soft theorem makes it impossible to set $A_{z}^{(0)}=D_{z} \varphi^{(0)}$ to zero on all boundaries. Hence $\varepsilon^{(1)}$, as well as $\varepsilon^{(0)}$, is a large gauge transformation, and maps one vacuum to a physically inequivalent one.

\section{CELESTIAL CURRENT WARD IDENTITY}

It is illuminating to rewrite scattering amplitudes as correlation functions on the celestial sphere, adopting the compact notation [7] 
$\left\langle z_{n+1}, \ldots|\mathcal{S}| z_{1}, \ldots\right\rangle \rightarrow\left\langle\mathcal{O}_{E_{1}}^{(1)}\left(z_{1}, \bar{z}_{1}\right) \cdots \mathcal{O}_{E_{n}}^{(1)}\left(z_{n} \bar{z}_{n}\right)\right\rangle$.

In this context, we define the subleading soft photon current

$$
J_{\bar{z}}^{(1)}=\frac{4 \pi}{e^{2}}\left(\left.D_{\bar{z}} \varphi^{(1)}\right|_{\mathcal{I}_{+}^{+}}-\left.2 D_{\bar{z}} \varphi^{(1)}\right|_{\mathcal{I}_{-}^{+}}+\left.D_{z} \varphi^{(1)}\right|_{\mathcal{I}_{-}^{-}}\right),
$$

where we have used the antipodal matching

$$
\left.\varphi^{(1)}\right|_{\mathcal{I}_{-}^{+}}=\left.\varphi^{(1)}\right|_{\mathcal{I}_{+}^{-}}
$$

The subleading soft theorem then becomes $[18,19]$

$$
\begin{aligned}
& \left\langle J_{\bar{z}}^{(1)} \mathcal{O}_{E_{1}}^{(1)}\left(z_{1}, \bar{z}_{1}\right) \cdots \mathcal{O}_{E_{n}}^{(1)}\left(z_{n} \bar{z}_{n}\right)\right\rangle \\
& \quad=\sum_{k=1}^{n} \frac{-i Q_{k}}{E_{k}\left(\bar{z}-\bar{z}_{k}\right)^{2}} D^{\bar{z}_{k}}\left\langle\mathcal{O}_{E_{1}}^{(1)}\left(z_{1}, \bar{z}_{1}\right) \cdots \mathcal{O}_{E_{n}}^{(1)}\left(z_{n}, \bar{z}_{n}\right)\right\rangle .
\end{aligned}
$$

The Mellin transform to a conformal basis for particles with helicity $s$ with conformal weights

$$
(h, \bar{h})=\frac{1}{2}(\Delta+s, \Delta-s)=\frac{1}{2}\left(-E \partial_{E}+s,-E \partial_{E}-s\right)
$$

is simply

$$
\mathcal{O}_{(h, \bar{h})}(z, \bar{z})=\int d E E^{\Delta-1} \mathcal{O}_{E}^{(1)}(z, \bar{z})
$$

In this conformal basis, (23) becomes the current algebra relation

$$
\begin{aligned}
& \left\langle J_{\bar{z}}^{(1)} \mathcal{O}_{\left(h_{1}, \bar{h}_{1}\right)} \cdots \mathcal{O}_{\left(h_{n}, \bar{h}_{n}\right)}\right\rangle \\
& =-i \sum_{k} \frac{Q_{k}}{\left(\bar{z}-\bar{z}_{k}\right)^{2}} D^{\bar{z}_{k}}\left\langle\mathcal{O}_{\left(h_{1}, \bar{h}_{1}\right)} \cdots \mathcal{O}_{\left(h_{k}-\frac{1}{2}, \bar{h}_{k}-\frac{1}{2}\right)} \cdots \mathcal{O}_{\left(h_{n}, \bar{h}_{n}\right)}\right\rangle .
\end{aligned}
$$

This is the celestial representation of the subleading soft theorem.

The operators $\mathcal{O}$ which create spacetime particles in a conformal basis appearing in celestial amplitudes are in different types of representations - typically the continuous unitary principal series $\$$ than those we are accustomed to in standard 2D CFT. The corresponding amplitudes take a rather different form often involving delta functions on the sphere [20-23], which makes possible relations between amplitudes with shifted conformal weights. Relations of this general type were noted in the gravitational context in [24] and verified by Stieberger and Taylor [25] in some special cases. It would be of interest to examine (26) in explicit examples.
Finally, we note that integrating around a contour $C$ weighted by an antiholomorphic function $\varepsilon(\bar{z})$, the subleading soft theorem takes the alternate form

$$
\begin{aligned}
& \left\langle\oint_{C} \frac{d \bar{z}}{2 \pi i} \varepsilon(\bar{z}) J_{\bar{z}}^{(1)} \mathcal{O}_{\left(h_{1}, \bar{h}_{1}\right)} \cdots \mathcal{O}_{\left(h_{n}, \bar{h}_{n}\right)}\right\rangle \\
& =-i \sum_{k \in C} Q_{k}\left\langle\mathcal{O}_{\left(h_{1}, \bar{h}_{1}\right)} \cdots \partial_{\bar{z}_{k}} \varepsilon\left(\bar{z}_{k}\right) D^{\bar{z}_{k}} \mathcal{O}_{\left(h_{k}-\frac{1}{2}, \bar{h}_{k}-\frac{1}{2}\right)} \cdots \mathcal{O}_{\left(h_{n}, \bar{h}_{n}\right)}\right\rangle,
\end{aligned}
$$

where the sum is restricted to operators inside the contour.

\section{ACKNOWLEDGMENTS}

This work was funded partially by DOE Grant No. DESC0007870. E. H. is funded by NSF Grant No. 1745303. We are grateful to Laura Donnay, Slava Lysov, and Dan Kapec for useful correspondence, and to Monica Pate, Ana Raclariu, and Sabrina Pasterski for discussion and advice throughout this work.

\section{APPENDIX: ASYMPTOTIC EXPANSION}

This Appendix gives a few details of the large $r$ expansion about $\mathcal{I}^{+}$.

A massless scalar field has $\frac{1}{r}$ expansion near $\mathcal{I}^{+}$as

$$
\Phi(u, r, z, \bar{z})=\sum_{n=1}^{\infty} \frac{\Phi^{(n)}(u, z, \bar{z})}{r^{n}} .
$$

The matter currents

$$
j_{\mu}=i Q\left(\bar{\Phi} \partial_{\mu} \Phi-\Phi \partial_{\mu} \bar{\Phi}\right)
$$

fall off as

$$
j_{u} \sim \mathcal{O}\left(\frac{1}{r^{2}}\right), \quad j_{z}, j_{\bar{z}} \sim \mathcal{O}\left(\frac{1}{r^{2}}\right), \quad j_{r} \sim \mathcal{O}\left(\frac{1}{r^{3}}\right) .
$$

Finite energy flux and charge suggest the falloffs

$$
A_{u} \sim \mathcal{O}\left(\frac{1}{r}\right), \quad A_{z}, A_{\bar{z}} \sim \mathcal{O}(1), \quad A_{r} \sim \mathcal{O}\left(\frac{1}{r^{2}}\right)
$$

In order to consistently solve the Maxwell equations in $\nabla^{\mu} A_{\mu}=0$ gauge we must allow logarithmic falloffs in the gauge fields. This gives the expansion 


$$
\begin{aligned}
& A_{u}=\sum_{n=2}^{\infty} \frac{A_{u}^{(n)}}{r^{n}}+\sum_{m=1}^{\infty} \frac{\tilde{A}_{u}^{(m)}}{r^{m}} \log r \\
& A_{r}=\sum_{n=2}^{\infty} \frac{A_{r}^{(n)}}{r^{n}}+\sum_{m=2}^{\infty} \frac{\tilde{A}_{r}^{(m)}}{r^{m}} \log r \\
& A_{z}=\sum_{n=0}^{\infty} \frac{A_{z}^{(n)}}{r^{n}}+\sum_{m=1}^{\infty} \frac{\tilde{A}_{z}^{(m)}}{r^{m}} \log r \\
& A_{\bar{z}}=\sum_{n=0}^{\infty} \frac{A_{\bar{z}}^{(n)}}{r^{n}}+\sum_{m=1}^{\infty} \frac{\tilde{A}_{\bar{z}}^{(m)}}{r^{m}} \log r .
\end{aligned}
$$

Our gauge condition leaves unfixed gauge transformations of the form $\square \varepsilon=0$, among which are residual gauge transformations with falloff $\mathcal{O}\left(r^{-1}\right)$ which, like a radiative massless scalar field, have an arbitrary boundary dependence. We have used this freedom to set $A_{u}^{(1)}=0$.

The Maxwell equations $\nabla_{\mu} F^{\mu \nu}=e^{2} j^{\nu}$ in retarded coordinates, with $F_{\mu \nu}=\partial_{\mu} A_{\nu}-\partial_{\nu} A_{\mu}$, are

$$
\begin{aligned}
\left(\partial_{u}-\partial_{r}\right)\left(r^{2} F_{r u}\right)+\gamma^{z \bar{z}}\left(\partial_{\bar{z}} F_{u z}+\partial_{z} F_{u \bar{z}}\right) & =e^{2} r^{2} j_{u} \\
-\partial_{r}\left(r^{2} F_{r u}\right)+\gamma^{z \bar{z}}\left(\partial_{\bar{z}} F_{r z}+\partial_{z} F_{r \bar{z}}\right) & =e^{2} r^{2} j_{r} \\
r^{2}\left(\partial_{r}-\partial_{u}\right) F_{r z}-r^{2} \partial_{r} F_{u z}-\partial_{z}\left(\gamma^{z \bar{z}} F_{z \bar{z}}\right) & =e^{2} r^{2} j_{z} \\
r^{2}\left(\partial_{r}-\partial_{u}\right) F_{r \bar{z}}-r^{2} \partial_{r} F_{u \bar{z}}-\partial_{\bar{z}}\left(\gamma^{z \bar{z}} F_{\bar{z} z}\right) & =e^{2} r^{2} j_{\bar{z}},
\end{aligned}
$$

while the Lorenz gauge condition reads

$$
-\partial_{u}\left(r^{2} A_{r}\right)-\partial_{r}\left(r^{2} A_{u}-r^{2} A_{r}\right)+\gamma^{z \bar{z}}\left(\partial_{z} A_{\bar{z}}+\partial_{\bar{z}} A_{z}\right)=0 .
$$

Together these imply

$$
\mathcal{O}(\log r): 2 \partial_{u} \tilde{A}_{\bar{z}}^{(1)}-2 \partial_{\bar{z}} \tilde{A}_{u}^{(1)}=0
$$

$$
\mathcal{O}(1):-2 \partial_{u} \tilde{A}_{u}^{(1)}=e^{2} j_{u}^{(2)}
$$

$$
2 \partial_{u} A_{\bar{z}}^{(1)}-2 \partial_{u} \tilde{A}_{\bar{z}}^{(1)}+2 D^{z} D_{z} A_{\bar{z}}^{(0)}=e^{2} j_{\bar{z}}^{(2)} .
$$

where we have used that $\mathcal{O}(\log r)$ expression for $\tilde{j}_{\bar{z}}^{(2)}$ is set to zero because the currents should not have logarithmic falloff. Note that $j_{u}^{(2)}$ would be incorrectly set to zero if $\log$ terms were not included in the expansion. We substitute (A8) and (A.9) into (A.10) to arrive at (12).
[1] A. Strominger, Asymptotic symmetries of Yang-Mills theory, J. High Energy Phys. 07 (2014) 151.

[2] T. He, P. Mitra, A. P. Porfyriadis, and A. Strominger, New symmetries of massless QED, J. High Energy Phys. 10 (2014) 112.

[3] D. Kapec, M. Pate, and A. Strominger, New symmetries of QED, Adv. Theor. Math. Phys. 21, 1769 (2017).

[4] C. Cheung, A. de la Fuente, and R. Sundrum, 4D scattering amplitudes and asymptotic symmetries from 2D CFT, J. High Energy Phys. 01 (2017) 112.

[5] A. Nande, M. Pate, and A. Strominger, Soft factorization in QED from 2D Kac-Moody symmetry, J. High Energy Phys. 02 (2018) 079.

[6] D. Kapec, M. Perry, A.-M. Raclariu, and A. Strominger, Infrared divergences in QED revisited, Phys. Rev. D 96, 085002 (2017).

[7] A. Strominger, Lectures on the Infrared Structure of Gravity and Gauge Theory (Princeton University Press, Princeton, NJ, 2018).

[8] F. E. Low, Scattering of light of very low frequency by systems of Spin 1/2, Phys. Rev. 96, 1428 (1954).

[9] M. Gell-Mann and M. L. Goldberger, Scattering of lowenergy photons by particles of Spin 1/2, Phys. Rev. 96, 1433 (1954).
[10] F. E. Low, Bremsstrahlung of very low-energy quanta in elementary particle collisions, Phys. Rev. 110, 974 (1958).

[11] T. H. Burnett and N. M. Kroll, Extension of the Low Soft-Photon Theorem, Phys. Rev. Lett. 20, 86 (1968).

[12] S. Weinberg, The Quantum Theory of Field. Vol 1: Foundations (Cambridge University Press, Cambridge, England, 2005).

[13] V. Lysov, S. Pasterski, and A. Strominger, Low's Subleading Soft Theorem as a Symmetry of QED, Phys. Rev. Lett. 113, 111601 (2014).

[14] M. Campiglia and A. Laddha, Subleading soft photons and large gauge transformations, J. High Energy Phys. 11 (2016) 012.

[15] E. Conde and P. Mao, Remarks on asymptotic symmetries and the subleading soft photon theorem, Phys. Rev. D 95, 021701(R) (2017).

[16] Just as in the case of the leading soft photon theorem in massless QED [2], which generalizes directly to massive QED [3,17], we expect our results to hold when we generalize to massive particles with trajectories that reach future timelike infinity $i^{+}$. For fields with spin there will be additional terms in the subleading soft factor, which for the identities considered herein become contact terms that vanish after contour integration. 
[17] M. Campiglia and A. Laddha, Asymptotic symmetries of QED and Weinberg's soft photon theorem, J. High Energy Phys. 07 (2015) 115.

[18] Up to contact terms which will vanish after contour integration.

[19] Using for a scalar operator that $-i E_{k} \mathcal{O}_{E_{k}}^{(2)}=\gamma^{z \bar{z}} D_{\bar{z}} D_{z} \mathcal{O}_{E_{k}}^{(1)}$, the right-hand side can be rewritten as $\frac{-Q_{k} \mathcal{O}_{E_{k}}^{(2)}\left(z_{k}, \bar{z}_{k}\right)}{\left(\bar{z}-\bar{z}_{k}\right)}$. This suggests a connection with the identification in [14] of subleading soft symmetries with gauge transformations that diverge linearly with $r$. It would be interesting to understand this better.

[20] S. Pasterski and S.-H. Shao, Conformal basis for flat space amplitudes, Phys. Rev. D 96, 065022 (2017).
[21] S. Pasterski, S.-H. Shao, and A. Strominger, Gluon amplitudes as $2 \mathrm{~d}$ conformal correlators, Phys. Rev. D 96, 085006 (2017).

[22] A. Ø. Schreiber, A. Volovich, and M. Zlotnikov, Tree-level gluon amplitudes on the celestial sphere, Phys. Lett. B 781, 349 (2018).

[23] S. Stieberger and T. R. Taylor, Strings on celestial sphere, Nucl. Phys. B935, 388 (2018).

[24] L. Donnay, A. Puhm, and A. Strominger, Conformally soft photons and gravitons, J. High Energy Phys. 01 (2019) 184.

[25] S. Stieberger and T. R. Taylor, Symmetries of celestial amplitudes, Phys. Lett. B 793, 141 (2019). 